> Contrairement aux choix effectués dans d'autres pays, ce n'est que récemment que l'évaluation des actions de santé a intégré une dimension économique de manière explicite en France. II existe pourtant depuis toujours des limites dans les ressources que l'on accepte de consacrer collectivement à la santé. Une évaluation économique comparative est dès lors indispensable pour orienter les choix de santé et contribuer à la régulation des prix. Les méthodologies reposent sur l'estimation de ratios différentiels coût/résultats comparés à une référence. L'interprétation de ces ratios n'est jamais isolée du contexte de la décision. L'évaluation économique en santé ne participe pas du rationnement des soins, mais elle contribue à la rationalité des décisions dans l'intérêt de tous. <

L'évaluation sert la décision, éclaire les choix et limite l'arbitraire. Des décisions, la plupart des acteurs du système de santé doivent en prendre régulièrement, à commencer par les responsables politiques du secteur. L'évaluation a ainsi peu à peu pénétré tous les domaines de la santé. Qu'il s'agisse de compétences, de pratiques, de produits (médicaments ou dispositifs) ou de stratégies préventives ou curatives, l'évaluation s'inscrit depuis longtemps dans une logique prédictive (ex-ante), formative ou sommative comme une composante essentielle des démarches médicales et sociales, du moins dès que ces dernières dépassent le simple choix individuel.

En France, ce n'est que très récemment que cette évaluation des actions de santé a intégré une dimension économique, du moins de manière explicite.

Jusque là, les questions financières dans le champ de la santé étaient jugées irritantes ou renvoyées à un futur hypothétique. Les politiques en appelaient à la responsabilité économique des médecins et à leur sens moral. Les médecins jugeaient inestimables leurs interventions ; les malades souhaitaient bénéficier sans contrainte des innovations particulièrement rapides

\section{L'évaluation économique dans le champ de la santé}

Bruno Detournay

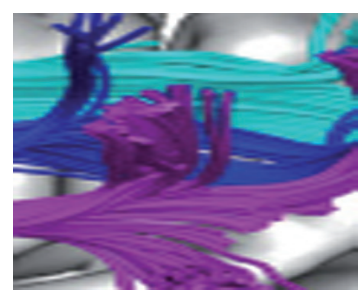

CEMKA-EVAL, 43, boulevard du Maréchal Joffre, 92340 Bourg-la-Reine, France. bruno.detournay@cemka.fr

dans ce domaine. La croissance relativement continue de la richesse collective permettait d'espérer dégager sans cesse de nouvelles ressources à consacrer à un secteur économique par ailleurs florissant en termes de profits et d'emplois.

$\varepsilon n$ reconnaissant l'existence d'un droit fondamental aux soins de santé, élargi à une notion de bien-être global, et en ayant adopté (au-delà des dispositifs caritatifs d'assistance aux plus démunis) des systèmes de santé reposant sur un financement collectif obligatoire, les États modernes avaient estimé résoudre un des aspects les plus dérangeants de la question sanitaire, à savoir celui de l'équité face à la maladie.

Dans un tel contexte, les remarques des économistes souvent considérés comme de simples comptables rationnaires n'étaient guère entendues. Bien sûr, les performances comparées des systèmes de santé internationaux n'étaient pas nécessairement en rapport avec les financements engagés, les inégalités de santé persistaient, le poids des charges sociales pesait sur d'autres secteurs de l'économie et la croissance des dépenses de santé était très supérieure à la croissance générale de l'économie. Des mesures étaient mises en œuvre face à ces constats, mais elles restaient souvent ponctuelles, ciblant tel ou tel aspect des dépenses, et sans véritable cohérence.

En France, une des réformes importantes introduites il y a presque vingt ans a été la reconnaissance officielle qu'il devait y avoir un plafond aux dépenses de santé, et que le niveau de ce plafond devait être défini par la représentation nationale. Mais les différents ONDAM (objectifs nationaux de dépenses d'assurance maladie) n'ont jamais été respectés depuis cette date. Surtout, le raisonnement ayant concouru à leur création n'a pas été poussé à son terme. 


\section{Pourquoi une évaluation économique?}

Faut-il mettre en place tel ou tel programme de santé publique plutôt qu'un autre? Faut-il accepter de prendre en charge collectivement tel ou tel traitement coûteux dans le cadre budgétaire défini ? Quel est le montant qu'il est raisonnable de payer pour bénéficier d'une nouvelle technologie de santé quand les mécanismes du marché ne s'appliquent pas pour fixer le prix de cette dernière (prescripteur non payeur, absence de concurrence, asymétrie d'information, etc.) ? Quel budget faudra-t-il dégager pour assurer le financement de ce traitement? Voilà les questions appelant la nécessité de conduire des évaluations économiques en santé.

On peut certainement débattre des niveaux de cotisations ou d'impôts affectés au domaine de la santé, ou contester les arbitrages politiques dans la répartition des moyens entre différents secteurs relevant de l'action publique. Quels que soient ces niveaux et ces arbitrages, l'expérience montre qu'il existe toujours des limites dans les ressources que l'on accepte de consacrer collectivement à la santé.

Ces ressources sont consommées par la mise en œuvre des interventions en santé, que ces dernières soient préventives ou curatives, diagnostiques ou palliatives. Dans sa conduite, l'action de santé mobilise des moyens humains et matériels qui ne sont plus alors utilisables pour autre chose. Dans le même temps, elle se traduit par un ensemble de résultats positifs et négatifs qui ont eux-mêmes des conséquences économiques. La reconnaissance de l'intérêt d'une action de santé soulève donc inévitablement une série de questions économiques à laquelle nous ne pouvons échapper, sauf à considérer que «la santé n'a pas de prix ».

Que l'on ne s'y trompe pas. La mesure de l'efficacité médicale reste évidemment le critère premier dans ces décisions. II y a là un principe éthique et médical qui figure déjà implicitement dans le serment d'Hippocrate «Dans quelque maison que je rentre, j'y entrerai pour l'utilité des malades [...] » [1]. Mais on doit alors mettre en regard de cette efficacité attendue ou observée les implications économiques de l'action, par exemple en matière de ressources financières ou humaines mobilisées au détriment d'autres choix possibles.

L'évaluation médicale prend alors une dimension économique. Elle contribue à orienter les priorités de santé, mais elle a également un rôle essentiel dans la régulation des prix, des produits et des services de santé, en éclairant les pouvoirs publics sur le rapport entre la valeur ajoutée attendue de ceux-ci au regard des prix souhaités par les industriels. Elle se substitue de la sorte aux mécanismes du marché souvent inopérants dans ce champ particulier.

\section{Quelles méthodes?}

Deux approches principales sont mises en œuvre en matière d'évaluation économique en santé. Sous la contrainte d'un budget défini, un optimum de quantité de résultats est obtenu en choisissant les interventions qui présentent le meilleur ratio différentiel coût/résultats (ou ratio d'efficience) à concurrence du budget disponible. Une autre possibilité passe par la fixation d'un seuil de référence qui permet de retenir les actions dont le ratio coût/résultats est inférieur à ce seuil et d'exclure les autres mais, dans ce cas, il n'y a plus d'encadrement budgétaire global. En pratique, il est possible de combiner ces deux approches en faisant varier le seuil de référence fixé a priori en fonction du degré de réalisation des objectifs budgétaires. Ces approches supposent que ces résultats de santé soient exprimés sur la base d'une unité universelle permettant d'arbitrer entre les actions de santé. La plus simple de ces unités correspond à l'année de vie gagnée traduisant l'espérance de gain de survie liée à un traitement qui réduirait la mortalité. Toutes les interventions en santé ne permettant pas de sauver des vies, ce critère n'est donc pas suffisant. Les économistes ont proposé de le compléter par le concept de PALY (quality adjusted life years). Les gains de santé sont alors mesurés en termes d'années de vie, pondérées par leur qualité : chaque temps de vie se voit affecter un cœfficient compris entre 0 (état de mort) et 1 (parfaite santé) exprimant toutes les gradations de mal-être envisageables. L'intégration des temps passés dans les différents états de santé une fois pondérés constitue le résultat de santé mesuré. Cette solution, souvent critiquée pour des raisons méthodologiques, a le mérite de la simplicité.

L'estimation du ratio d'efficience suppose également de disposer d'une évaluation des coûts différentiels entre les stratégies comparées. Cette évaluation se heurte à nombre de difficultés, car le coût d'un bien n'est pas une mesure intangible [2]. Le coût dépend du point de vue adopté, des éléments pris en compte pour le définir, des conventions de calcul retenues, du moment auquel il est mesuré, etc. Dans le cadre de l'évaluation économique, le terme de coût renvoie, d'une part, aux ressources consommées dans la production d'une intervention de santé et, d'autre part, aux ressources non consommées, mais rendues indisponibles par la production de l'intervention [3]. Ces quantités doivent ensuite faire l'objet d'une valorisation, c'est-à-dire d'une traduction en unités monétaires. En l'absence de prix établis par le marché, la valorisation est effectuée à partir des coûts de production, voire des tarifs administrés, car ils représentent de fait un prix reconnu par la collectivité [3].

Les coûts et bénéfices d'une intervention peuvent survenir à des temps différents, parfois très éloignés. Une dépense immédiate en matière de prévention, par exemple, peut éviter des dépenses futures sur le plan des traitements. Préférence pure pour le présent (on préfère toujours dépenser plus tard et gagner maintenant), effet richesse (les générations futures pourront produire davantage avec les mêmes ressources sous 
l'effet du progrès technique), prise en compte des risques associés à l'incertitude sur l'avenir constituent autant d'éléments à prendre en considération pour actualiser (ramener à une valeur présente) les coûts et bénéfices différés dans le temps.

Sur ces bases, il est possible d'évaluer l'efficience d'une intervention en santé en retenant deux méthodes principales : l'analyse coût/efficacité (où le critère d'efficacité est une mesure médicale si possible générique, c'est-à-dire applicable pour différentes interventions de santé telles que les années de vie gagnées) et l'analyse coût/utilité (où le critère d'efficacité est l'année de vie pondérée par sa qualité). Le choix de la méthode à privilégier dépend de la nature des conséquences attendues des interventions étudiées sur la santé au sens large des populations visées.

Quelle que soit la méthode d'évaluation retenue, cette dernière contient une part d'incertitude, d'imprécision, voire de controverse méthodologique à l'égal des évaluations uniquement médicales, même lorsque ces dernières reposent sur des approches éprouvées. L'estimation de l'incertitude associée aux conclusions de l'évaluation est donc nécessaire pour aboutir à un avis médical et économique sur une intervention. La mise en œuvre d'analyses de sensibilité permet alors de déterminer la robustesse des conclusions, comme d'identifier les facteurs dominants influant sur le résultat.

\section{Quelle interprétation?}

Une fois le ratio d'efficience établi pour l'ensemble des actions envisageables (et son incertitude caractérisée), un classement peut être établi selon ce critère. Les coûts induits par la mise en œuvre des interventions les mieux classées sont ensuite additionnés à concurrence des financements disponibles. Si l'on est contraint par un budget donné, c'est en retenant cette démarche qu'on peut maximiser les résultats de santé qui pourraient être obtenus au niveau de la population.

En pratique, il est très difficile de disposer de ce type d'information de manière exhaustive compte tenu de la multiplicité des interventions en santé. Pour cette raison, certains pays préfèrent fixer un seuil d'acceptabilité pour le ratio différentiel coût/résultats. De nombreuses réflexions sont en cours sur la manière de définir un tel seuil. La méthode la plus démocratique est sans doute celle qui recourt à l'estimation de la propension à payer des individus dans la collectivité pour un gain de santé donné. Une importante méta-analyse d'études de cette nature a été conduite récemment par l'OCDE (Organisation de coopération et de développement économique) [4] sur la base d'une revue systématique des publications internationales (près de 900 études analysées). Ce rapport situe la propension à payer en moyenne pour sauver une vie d'adulte dans les pays de l'OCDE entre 1,5 et 4,5 millions de dollars américains (USD de 2005), avec une valeur de référence de 3 millions d'USD. Les analyses des facteurs explicatifs des choix dans ce domaine montrent que ceux-ci sont surtout orientés par le niveau de richesse du pays considéré. Ces résultats ont été repris en France pour définir comme valeur tutélaire une estimation de la valeur statistique de l'année de vie gagnée fixée à $115000 €[5]$ dans le cadre des investissements publics. Aucune valeur de référence n'a, à ce jour, été définie en France en ce qui concerne les analyses de type coût/utilité. La responsabilité de l'interprétation des résultats repose donc, pour le moment, sur des bases non explicites faisant appel à différentes sources (ratios publiés pour différentes interventions actuellement financées, comparaisons internationales, etc.).

Au-delà de son apport dans la décision de mise à disposition d'un bien ou d'un service de santé, il est clair que l'évaluation économique conduite «a priori » peut constituer un élément important dans la négociation des prix entre l'administration et les producteurs. Ces derniers ont naturellement tendance à maximiser leur profit et à justifier une demande de prix élevés sur la base d'arguments difficiles à apprécier (importance des investissements de recherche, risque d'échec supporté, etc.). Marquer une limite dans ces ambitions à travers un raisonnement fondé sur une estimation de la valeur ajoutée d'un médicament, dispositif ou matériel pour un prix donné peut contribuer à modérer ces ambitions dans un contexte économique que chacun sait moins favorable que par le passé.

Dans tous les cas, le calcul économique autour d'une décision en santé ne fait pas à lui seul cette dernière. Il ne constitue qu'un élément d'orientation, contribuant à l'éclairage des responsables et s'inscrivant dans le cadre de l'évaluation générale de l'action. D'autres considérations relevant d'aspects éthiques, de la recherche d'une meilleure équité, voire de politique industrielle ou des souhaits exprimés par la population peuvent être légitimes pour modifier l'interprétation de l'évaluation économique, particulièrement quand les résultats de cette dernière restent incertains.

\section{Conclusion}

En matière de santé, la très grande hétérogénéité des pratiques, la complexité des choix à effectuer et l'importance des enjeux économiques, sociaux et éthiques inciteraient à une utilisation intensive du calcul économique dans l'aide à la décision publique [5]. Jusqu'à très récemment, l'administration et le corps médical se montraient assez réticents à prendre en compte cette approche.

Cette situation est sans doute en train d'évoluer. Différents textes législatifs successifs ont confié à la Haute autorité de santé depuis 2008 une mission en matière d'évaluation médico-économique sur les actes, produits ou prestations de santé [6]. Cette mission concerne, en particulier, l'évaluation économique de certains 
médicaments et dispositifs médicaux souhaitant être inscrits sur la liste des produits remboursables par l'Assurance maladie obligatoire [7]. Les avis émis doivent orienter les décisions de remboursement et les négociations de prix conduites par le Comité économique des produits de santé.

On aboutit ainsi, peu à peu, à l'idée d'un «panier de soins solidaire » ayant vocation à être accessible à tous sans barrière financière [8]. Les interventions candidates à figurer dans ce panier sont toutes les actions de santé présentant un rapport bénéfice/risque favorable sur le plan médical et ayant un ratio coût/résultats suffisant pour autoriser un financement par le système de santé national. Les prises en charge se situant hors de ce panier s'inscrivent alors, soit dans le cadre de la recherche médicale (et seraient éventuellement financées à ce titre), soit comme relevant de choix individuels et donc financées uniquement par les bénéficiaires de ces prises en charge.

L'évaluation économique en santé n'est donc pas un instrument participant du rationnement des soins. Elle vise, au contraire, à améliorer la rationalité de décisions jusqu'ici parfois incohérentes et purement financières, et à garantir un juste usage des ressources disponibles dans l'intérêt de tous. En ce sens, elle s'inscrit comme un progrès sur le plan de la justice sociale et de la protection des plus faibles. $\diamond$

\section{SUMMARY}

\section{Economic evaluation in healthcare}

Economics was only recently considered as a dimension of health technology assessment in France. Yet there are always limits in resources that we collectively agree to devote to health. A comparative "economic" appraisal is therefore needed to guide health choices and contribute to price regulation. Methodologies are based on the determination of incremental cost-effectiveness ratios compared with a reference. The interpretation of these ratios is never isolated from the context of the decision. Health economic evaluation is not involved in healthcare rationing, but it contributes to the rationality of decisions in the interest of the overall population. $\diamond$

\section{LIENS D'INTÉRÊT}

L'auteur, consultant, déclare avoir bénéficié de subventions et de contrats d'études ou d'expertise de la part de multiples entreprises privées et institutions publiques nationales ou internationales.

\section{RÉFÉRENCES}

1. Hippocrate. Serment d'Hippocrate. In Fuuvres complètes d'Hippocrate, traduction et commentaires d'E Littré. Paris : édition J.B. Baillière et fils, 1861.

2. Riveline C. Évaluation des coûts. Éléments d'une théorie de la gestion. Paris : Les Presses de l'École des Mines, 2005.

3. HAS. Guide méthodologique. Choix méthodologiques pour l'évaluation économique à la HAS. HAS, 2011.

4. OCDE. Mortality risk valuation in environment, health and transport policies. OECD Publishing, 2012.

5. Commissariat général à la stratégie et à la prospective. L'évaluation socio-économique des investissements publics. Rapport de la Commission présidée par $\varepsilon$. Quinet, septembre 2013.

6. Code de la Sécurité sociale. Article L161-37.

7. Décret $n^{\circ} 2012-1116$ du 2 octobre 2012 relatif aux missions médicoéconomiques de la Haute autorité de santé. JORF 4 octobre 2012; 0231 : 15522 .

8. Askenazy P, Dormont B, Geoffard Py, Paris V. Pour un système de santé plus efficace. Les Notes du Conseil d'Analyse Économique 2013 ; 8 .

\section{TIRÉS À PART}

B. Detournay

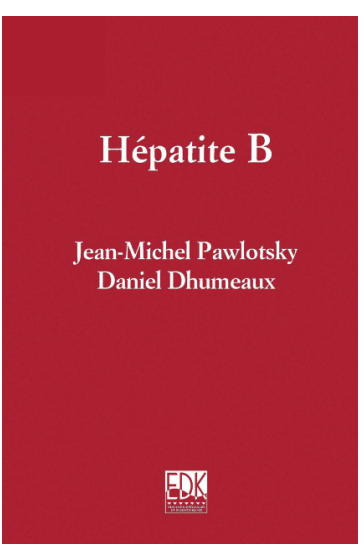

ISBN : 978-2-8425-4131-6 576 pages

\section{Bon de commande}

À retourner à EDK, 2, rue Troyon - 92316 Sèvres Cedex

Tél. : 0155641393 - Fax : 0155641394 - E-mail : edk@edk.fr

NOM :

Prénom :..

Adresse :

Code postal :

Ville :

Pays :

Fonction :

Je souhaite recevoir l'ouvrage Hépatite B : $54 €+3 €$ de port $=\mathbf{5 7} €$ TTC offre exceptionnelle réservée aux abonnés à $\mathrm{m} / \mathrm{s}$ jusqu'au 31 décembre 2010

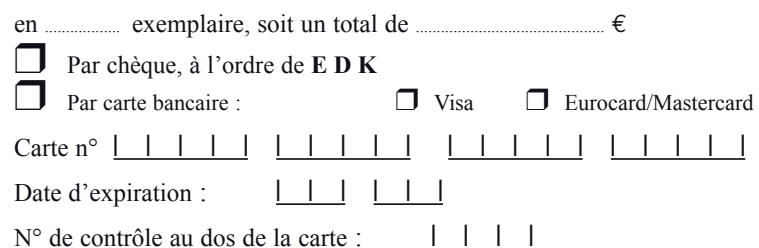

Signature : 